\title{
Multipath Polyphase Circuits and their Application to RF Transceivers
}

\author{
Eric Klumperink, Rameswor Shrestha, Eisse Mensink, Gerard Wienk, Zhiyu Ru, Bram Nauta \\ IC Design group, CTIT, University of Twente, Enschede, The Netherlands
}

\begin{abstract}
Nonlinearity and time-variance in Radio Frequency (RF) circuits leads to unwanted harmonics and intermodulation products, e.g. in power amplifiers and mixers. This paper reviews a recently proposed multipath polyphase circuit technique which can cancel such harmonics and intermodulation products. This will be illustrated using a power upconverter IC as an example. The upconverter works from DC to $2.4 \mathrm{GHz}$, and the multipath polyphase technique cleans its spectrum up to the 17 th harmonic, keeping unwanted spurious responses more than $40 \mathrm{~dB}$ below the carrier. The technique can also be useful for other applications, and some possible applications will be discussed.
\end{abstract}

\section{INTRODUCTION}

Wireless radio communication has recently experienced an enormous growth, pushing radio transceiver technologies to use scarce available radio spectrum as effective as possible. Nonlinearity and time-variance in RF transceiver circuits are two important RF impairments resulting in non-optimal use of radio spectrum, as they cause spectral components interfering with other radio channels. Fig.1 illustrates this point, taking a radio transmitter as an example. To produce significant output power, typically in the range between $1 \mathrm{~mW}$ to $10 \mathrm{~W}$, power drivers and power amplifier circuits with transistors working at large signal swings are used. Thus non-linearity of the transistors plays an important role, resulting in harmonics (see Fig.1) and intermodulation distortion products at many unwanted frequencies [1]. As the power efficiency of most amplifiers increases for higher signal swings, it is desirable to drive the amplifiers to a level close to their compression point.

Apart from nonlinearity, a time-variant transfer function can also introduce many unwanted frequency components. A hard-switched up-conversion mixer as shown Fig. 1 can be modeled as a linear time-variant circuit, with a linear transfer from the base-band (BB) input to RF-output, which changes sign instantaneously with the state of the local oscillator (LO) signal. If a square wave LO-signal switching with $50 \%$ duty cycle is assumed, the BB-signal is not only upconverted to $\omega_{\text {LO }}$, but also to unwanted higher odd harmonics with a relative strength of $1 / 3,1 / 5,1 / 7$, etc. compared to the fundamental. Thus the 9th harmonic is still stronger than $20 \mathrm{~dB}$ below the fundamental, which is often problematic.
In order to avoid this unwanted harmonic mixing, the input signal could be multiplied by a sine wave signal using a highly linear multiplier. However, realizing a linear multiplier is much more difficult than a hard-switched mixer, and the generation of a clean sine wave is also problematic, especially for a large frequency range. Typical sine-wave oscillators, e.g. LC oscillators have only a limited tuning range in the order of $5-50 \%$. If a larger tuning range is needed, digital dividers are commonly exploited to divide the VCO frequency. As digital circuits benefit from Moore's law, we strongly prefer flexible digital synthesizer techniques over analog sine wave generation. However, this means we have to find a solution to suppress unwanted harmonics.

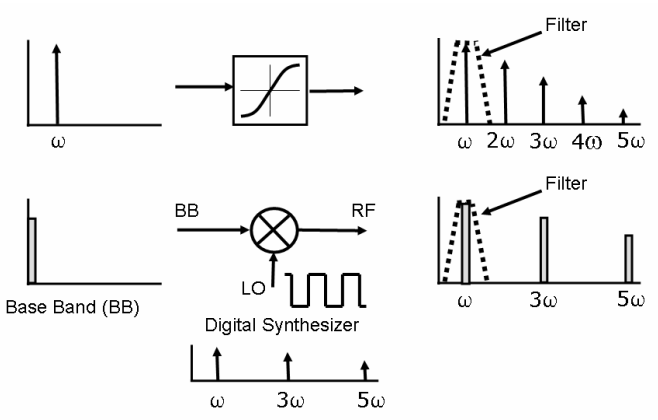

Figure 1. Both nonlinearity (e.g. power amplifier) and time-variance (e.g. hard-switched mixer) generate unwanted spectral components, which are traditionally removed by dedicated band-pass filters.

In traditional radio transmitters, these unwanted products are rejected using dedicated band-pass filters typically implemented using inductors and capacitors (LC filters). We like to avoid such filters, especially on CMOS chips, as they require high quality inductors which are difficult to implement and/or take large chip area. Especially when a flexible choice of the frequency is wanted, e.g. to realize a software defined radio platform, such filters are problematic as LC band-pass filters work at a fixed frequency related to the LC-resonance frequency, which limits the flexibility in choosing a TXfrequency. The next section reviews a polyphase multi-path technique [2][3] to eliminate these filters or relax their requirements significantly. In section III we will discuss a way to implement the different phases using mixers, and in section IV an example of a power up-converter exploiting the 
technique. Section $\mathrm{V}$ discusses alternative application directions and section VI draws conclusions.

\section{Polyphase Multi-Path Circuits for Spectral PURITY ENHANCEMENT}

Fig.1 shows a nonlinear circuit excited by a single sine wave at $\omega$, producing a wanted output signal at $\omega$ but also unwanted harmonic distortion at $2 \omega, 3 \omega, 4 \omega$, etc.. Fig. 2 shows a polyphase 3-path circuit, cancelling many harmonics of $\omega$ [2]. The basic idea is to divide the nonlinear circuit of Fig.1 into ' $n$ ' equal smaller pieces, and apply an equal but opposite phase shift before and after each nonlinear circuit. If the phase shift in path ' $\mathrm{i}$ ' is $(\mathrm{i}-1) \times \varphi$, where $\varphi$ is a phase shift constant satisfying $n \times \varphi=360^{\circ}$, the circuit will produce the same fundamental tone as in Fig.1, but cancel many harmonics. Mathematically this can be shown using a power series expansion, assuming a memory-less weakly nonlinear system. If the signal $x(t)=A \cos (\omega t)$ is applied to the input, the output of the nonlinear circuit of the ith path can be written as:

$$
\begin{aligned}
p_{i}(t)=a_{0}+ & a_{1} \cos (\omega t+(i-1) \varphi) \\
+ & a_{2} \cos (2 \omega t+2(i-1) \varphi) \\
& +a_{3} \cos (3 \omega t+3(i-1) \varphi)+\ldots \ldots .
\end{aligned}
$$

Where $a_{0}, a_{1}, a_{2}, a_{3} \ldots$. are Taylor series constants characterizing the nonlinearity [1]. From equation (1), it can be seen that the phase of the ' $\mathrm{k}$ 't, harmonic at the output of the nonlinear circuit rotates by ' $k$ ' times the input phase (i-1) $\varphi$. The phase shifters, -(i-1) $\varphi$, after the nonlinear blocks are required to align the fundamental components at $\omega$ in phase again. The signals at the output of these phase shifters can be written as:

$$
\begin{aligned}
y_{i}(t)=a_{0}+ & a_{1} \cos (\omega t) \\
+ & a_{2} \cos (2 \omega t+(i-1) \varphi) \\
& +a_{3} \cos (3 \omega t+2(i-1) \varphi)+\ldots \ldots . .
\end{aligned}
$$

In equation (2), the phase of the fundamental component is identical for all the paths, but the phases of the harmonics are different for each path. If the phase $\varphi$ is chosen such that $\varphi=360^{\circ} / \mathrm{n}$, then all the higher harmonics are cancelled [2], except for the $\mathrm{k}^{\text {th }}$ harmonics for which $\mathrm{k}$ equals $\mathrm{j} \times \mathrm{n}+1 \quad \mathrm{j}=0,1$, $2,3, .$.$) .$

The simplest example of a polyphase multi-path circuit is a well-known differential circuit driven with balanced (antiphase) input signals. It cancels all even harmonics (no cancellation of $\mathrm{k}=\mathrm{j} \times 2+1$, i.e. odd harmonics).

A system with three paths is shown in Fig.2. In this case, phase shifts of $0^{\circ}, 120^{\circ}$ and $240^{\circ}$ are added before the nonlinear block to path 1, 2 and 3 respectively, and equal but opposite phases $-0^{\circ},-120^{\circ}$ and $-240^{\circ}$ behind the block. Due to the nonlinearity, the phase rotation for the $\mathrm{k}^{\text {th }}$ harmonic is $\mathrm{k}$ times the input phase. Thus the respective phases at the output of the nonlinear block for path $[1,2,3]$ are $\left[0^{\circ}, 120^{\circ}, 240^{\circ}\right]$ for $\omega,\left[0^{\circ}, 240^{\circ}, 120^{\circ}\right]$ for $2 \omega$ and $\left[0^{\circ}, 0^{\circ}, 0^{\circ}\right]$ for $3 \omega$ products. Fig.2 also shows how the phases of the harmonics at the output of each path combine. Only the fundamental components add up in phase ( $\omega$-arrows), while the vectors for the second and third harmonics create a "balanced structure"

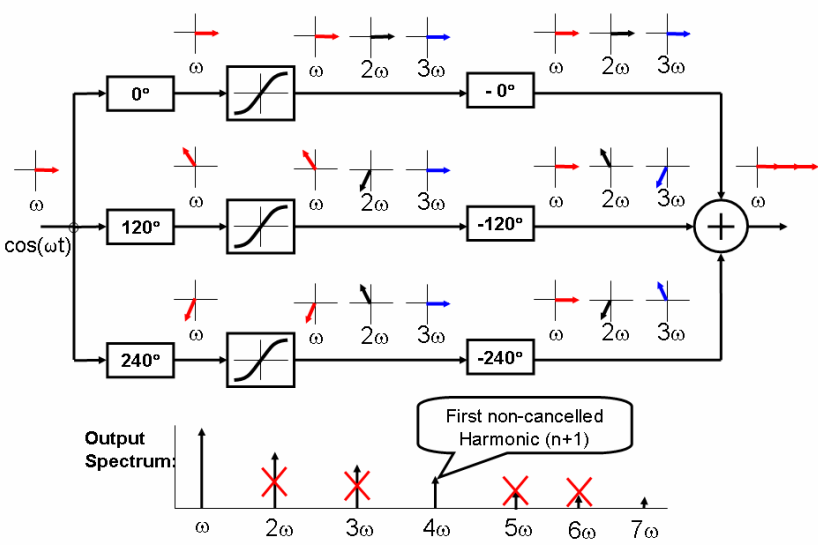

Figure 2. Polyphase 3-path circuit with harmonic cancellation except for harmonics $\mathrm{j} \times \mathrm{n}+1$ (for $\mathrm{n}=3$, harmonics $1,4,7, \ldots$ are not cancelled).

at the output, resulting in a zero sum (cancellation). However, the fourth harmonic components will align in phase again, and will add up like the fundamental. The output spectrum in the lower part of Fig.2 shows that the 2nd, 3rd, 5th, 6th etc harmonics are cancelled and the first non-cancelled is the fourth for a 3-path system. Similarly for a 4-path system the first non-cancelled harmonic will be the fifth harmonic and in general for an n-path system the $(\mathrm{n}+1)$ th harmonic is the first non-cancelled harmonic. Theoretically, an infinite number of paths is needed to cancel all the harmonics. However, in practice higher order harmonics are weaker than low order harmonics and need not all be cancelled. Also, some filtering will in practice always be present, e.g. due to the limited bandwidth of an antenna or the speed limitations in a circuit. Moreover mismatches will put a practical limit on what is feasible [2].

If the non-linear system is excited by a two-tone input signal $x(t)=A_{1} \cos \omega_{1} t+A_{2} \cos \omega_{2} t$, besides harmonics the output will also contain intermodulation products at new frequencies $p \omega_{1}+q \omega_{2}$, where $p$ and $q$ identify harmonics of $\omega_{1}$ and $\omega_{2}$ respectively, and can be positive or negative integer numbers. It can be shown [2] that most intermodulation products are cancelled, except if $\mathrm{p}+\mathrm{q}$ equals $\mathrm{j} \times \mathrm{n}+1$ (where $\mathrm{j}=0,1,2,3, \ldots)$.

\section{MIXER: PHASE AND FREQUENCY SHIFTER}

To realize wideband harmonic rejection using a polyphase multi-path system, we need very wideband phase shifters before and after the nonlinearity. This is because all phase shifters need to have a constant phase shift over all relevant frequencies involved in the cancellation process. In a DSP intensive radio transmitter, digital signal processing techniques can be exploited to realize phase shifters before D/A conversion and nonlinear power amplification. Therefore, a good solution can be to shift this polyphase generation problem to the digital domain, and use a DSP followed by multiple DACs to generate multi-phase baseband signals. However, behind the nonlinear element we are in the analog domain, and there can be many harmonics. In that case cancellation of a multitude of harmonics requires a constant phase shift over many octaves of frequency.

A very wideband phase shifter can be implemented with a mixer, since a mixer as shown in Fig.1 transfers phase 
information of both the "baseband" (BB) and "Local Oscillator" (LO) port to the output. Whatever phase is added to the LO signal will appear at the output of the mixer. So by replacing the second set of phase shifters in Fig.2 with mixers, as shown in Fig.3, we can achieve a wideband phase shift but simultaneously we will get frequency conversion.

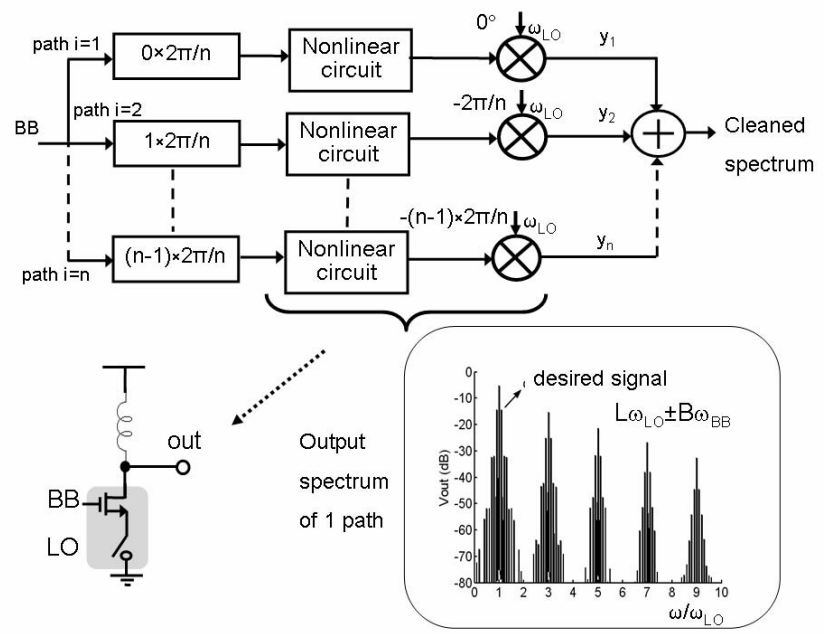

Figure 3. Polyphase n-path transmitter using mixers as phase shifters, where each path can be as simple as a transistor with switch. Due to timevariance (switching) and nonlinearity the output spectrum for 1 path has many harmonics and sidebands, which can be cancelled using $\mathrm{n}$ paths.

As upconversion is desired in a transmitter circuit anyway, this fits nicely to our goal. However, a mixer produces not only a sum frequency but also a difference frequency. Usually only one of these is the wanted signal, while the other ("the image") needs to be suppressed. Moreover, the LO-signal usually is a square wave containing many harmonics, because flexible frequency synthesizers rely on digital dividers, as discussed in the previous section. For power efficiency reasons it is also highly desired to use a switching mixer and a large BB-signal swing, e.g. a single transistor with switch as shown in Fig.3. Thus, the output spectrum for one path will now contain a forest of harmonics and sidebands as shown in Fig.3 for the case with a single-tone BB-signal. Spectral components occurs at frequencies $\mathrm{L} \omega_{\mathrm{LO}} \pm \mathrm{B} \omega_{\mathrm{BB}}$, where $\mathrm{L}$ and $\mathrm{B}$ are integers, due to the multiplication of the square wave LO with the baseband input signal BB, and also the nonlinearity of the circuit. In the next section we will see how we can exploit the polyphase multipath technique to cancel almost all the unwanted components.

\section{FILTER-LESS POWER UP-CONVERTER}

A power upconverter combines the functionality of a power amplifier and upconversion mixer. The PA and mixer can be as simple as shown in Fig.3, which is equivalent to first amplification and then mixing. Here the PA is a single transistor operating as transconductor ( $\mathrm{V}-\mathrm{I}$ converter), which is switched on and off by the LO signal via a switch (NMOS transistor driven by a digital inverter). Thus the V-I conversion and upconversion is done in the same circuit, via a switched transconductor mixer [4]. With respect to efficiency this circuit resembles a single transistor (class A) power amplifier. However, due to the polyphase multipath technique distortion products are cancelled and larger signal swings can be tolerated, improving efficiency.

Unfortunately, a few problematic products remain still present at the output. Since we have two input ports now (BB and LO), and mixing produces several sum and difference frequencies, a slightly different condition for non-cancelled products is found $[10,11](\mathrm{L}=\mathrm{j} \times \mathrm{n}+\mathrm{B}$ where $\mathrm{j}=\ldots .-2,-1,0,1$, $2 \ldots$, and $\mathrm{B}$ is a positive or negative integer number). Especially the $3 \omega_{\mathrm{LO}}+3 \omega_{\mathrm{BB}}$ is troublesome because the $3 \mathrm{rd}$ order distortion term is usually much stronger than higher order distortion components [1] and is also close to the desired signal. It cannot be cancelled with any number of paths as all products for which $\mathrm{L}=\mathrm{B}$ are not cancelled $(\mathrm{j}=0$ case, so independent of $n)$. To eliminate the strong $3 \omega_{\mathrm{LO}}+3 \omega_{\mathrm{BB}}$ terms, the duty cycle of the LO was chosen to be 1/3 [3]. By doing so, the 3rd, 6th, 9th, etc harmonic terms disappear from the Fourier series expansion, however some even order terms appear. Fortunately, it is quite easy to cancel even order products by using a differential baseband input (balancing).

To demonstrate the feasibility of a highly flexible multipath transmitter, we designed a power upconverter in $0.13 \mu \mathrm{m} \mathrm{CMOS}$, covering all frequencies up to $2.4 \mathrm{GHz}$. To show wideband spectral cleaning we designed an 18-path system, which can clean-up the spectrum up to the 17th harmonic [3]. Fig.4 shows the output frequency spectrum for a transmit frequency of $350 \mathrm{MHz}$ for one path (no cancellation) and for the complete 18-path system (lower part of Fig.4). Clearly all problematic products are suppressed significantly. Please note that the unfortunate FM-radio spurs that are modulated with our output signal are caused by a $100 \mathrm{MHz}$ high power FM-radio broadcast transmitter on the roof of our building. Overall, 10 chips were measured with harmonic components $<-40 \mathrm{dBc}$ up to the 17 th harmonic of the LO, for an LO-frequency from $30-800 \mathrm{MHz}$. For higher frequencies the chip has a 6-path mode which was measured for $30 \mathrm{MHz}-$ $2.4 \mathrm{GHz}$ with similar rejection up to the fifth harmonic of the LO.

The multi-path technique cleans the output spectrum from unwanted harmonics, which result from the hard-switching mixer, but also from non-linearity in the switched transconductor. Simulations and measurements show that we can drive the power upconverter close to its $1 \mathrm{~dB}$ compression point with harmonics well below $-40 \mathrm{dBc}$. For $1.2 \mathrm{~V}$ supply voltage we can realize a $2.5 \mathrm{Vpp}$ output voltage swing directly over a differential $100 \Omega$ load, without impedance transformation, corresponding to $8 \mathrm{~mW}$ output power.

At this point it might be useful to compare the polyphase multipath technique to another well-known harmonic rejection mixer technique [5]. In [5], also multiple mixers driven with multi-phase square-wave LO signals are used, as in Fig.3. However, the phase shifters before the nonlinear blocks are missing and all mixers have the same input signal with the same phase. The harmonic rejection is achieved by weighting factors on mixer input signals (i.e. $1: \sqrt{ } 2: 1$ ) and 45 -degree phase difference between the LO phases to suppress the $3 \mathrm{rd}$ and 5th harmonic, to emulate a sinusoidal LO signal. In contrast, the polyphase multipath technique uses no amplitude weighing factor, but only phase shifts before and after a 
nonlinear element. The polyphase multipath technique not only addresses harmonic mixing, but also cancels harmonics produced by nonlinearities in the $\mathrm{BB}$ to $\mathrm{RF}$ transfer.

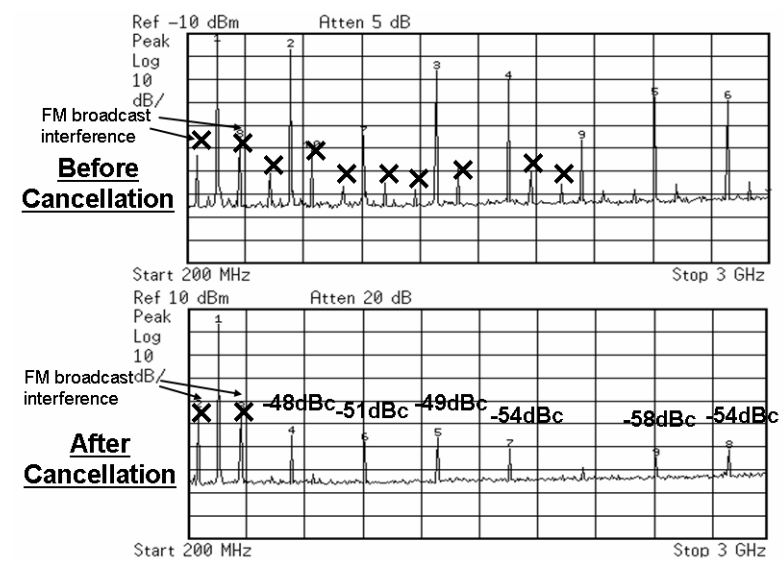

Figure 4. Output spectrum of one path ("before cancellation") and the sum-output of a 18-path power up-converter [3].

\section{OTHER APPLICATIONS}

Nonlinearity and time-variance does not only occur in radio transmitter circuits, but in many other circuits. Therefore the polyphase multipath technique is potentially useful for many other applications. Two examples relevant for RF transceivers will be discussed: an RF receiver and a frequency synthesizer.

In wideband receivers, e.g. for traditional analog $\mathrm{TV}$ and for new digital TV standard such as DVB-H, harmonic mixing is a problem because channels are spread over a wide input frequency range so that harmonics can be in the same band with the desired channel, or because other applications reside at frequencies which are harmonically related to the desired channel (e.g. an undesired $1800 \mathrm{MHz}$ GSM signal at the third harmonic of a $600 \mathrm{MHz}$ wanted TV-band signal) and pre-select filter cannot deliver sufficient suppression. Polyphase multipath circuits can be useful to attenuate unwanted harmonic mixing products. A possible implementation can be using multiple mixers driven with polyphase LO-signals to realize the first phase shifters in Fig.2, while the second phase shifters can be realized using a vector multiplier principle (weighted addition of a I and $\mathrm{Q}$ signal). Recently we discovered this idea of implementation has been developed independently by others [6] (based on a different principle of emulating a sinusoidal LO signal), and applied in a harmonic rejection mixer/tuner.

Frequency synthesis is another application field where polyphase multipath circuits may be used with advantage. In frequency synthesis systems, single sideband mixers are often used to generate the sum or difference frequency. This is for instance the case in Ultra Wide Band receivers which apply very fast frequency hopping (e.g. <10nsec hop time [7]). These hop-times are much too small for a typical Phase Locked Loop to settle. A solution is to synthesize the wanted frequencies as sums or differences of continuously available generator signals. E.g. for UWB roughly $3.5 \mathrm{GHz}, 4 \mathrm{GHz}$ and $4.5 \mathrm{GHz}$ are needed, which can be generated from $4 \mathrm{GHz}$ and $500 \mathrm{MHz}$ via single sideband mixers. Only one product is wanted in this case, which asks for a single-sideband mixer. However, often higher harmonics introduce unwanted spurious components in the frequency synthesizer output signal. A polyphase multipath circuit can operate as a singlesideband mixer with the added advantage of also suppressing such unwanted spurious components. This eliminates filters which are others needed [7]. Especially when ring oscillators are used, which often produce multiple phases of an oscillator signal, it can be rather straightforward to implement the polyphase multipath technique.

\section{CONCLUSIONS}

Nonlinear and/or time-variant behavior of radio transceiver circuits is useful to realize frequency conversion, but also produces many unwanted harmonics and sidebands. This paper reviewed a polyphase multi-path technique that rejects many of these unwanted products, without using any dedicated filters. Using this technique, a highly flexible power upconverter has been realized on in CMOS, operating at an arbitrary frequency between DC and $2.4 \mathrm{GHz}$, with unwanted harmonics and sideband lower than $-40 \mathrm{dBc}$ up to the 17 th harmonic. Also, we discussed potential other applications like circuits for radio receivers and frequency synthesis.

\section{ACKNOWLEDGEMENTS}

The authors would like to thank Fokke Hoeksema and Vincent Arkesteijn for their contributions to discussions on this work, and Henk de Vries for practical assistance during design and measurements. Philips Research is acknowledged for providing the silicon.

\section{REFERENCES}

[1] W. Sansen, "Distortion in elementary transistor circuits," Circuits and Systems II: Analog and Digital Signal Processing, IEEE Transactions on], vol. 46, pp. 315-325, 1999.

[2] E. Mensink, E. A. M. Klumperink, and B. Nauta, "Distortion cancellation by polyphase multipath circuits," Circuits and Systems I: Regular Papers, IEEE Transactions on, vol. 52, pp. 1785-1794, 2005.

[3] R. Shrestha, E. Mensink, E. A. M. Klumperink, G. J. M. Wienk, and B. Nauta, "A multipath technique for canceling harmonics and sidebands in a wideband power upconverter," 2006, IEEE International SolidState Circuits Conference (ISSCC), San Francisco, February 6-8, 2006. ISSCC Digest, pp. 452-453.

[4] E. A. M. Klumperink, S. M. Louwsma, G. J. M. Wienk, and B. Nauta, "A CMOS switched transconductor mixer," Solid-State Circuits, IEEE Journal of, vol. 39, pp. 1231-1240, 2004.

[5] J. A. Weldon, R. S. Narayanaswami, J. C. Rudell, L. Li, M. Otsuka, S. Dedieu, T. Luns, T. King-Chun, L. Cheol-Woong, and P. R. Gray, "A $1.75-\mathrm{GHz}$ highly integrated narrow-band CMOS transmitter with harmonic-rejection mixers," Solid-State Circuits, IEEE Journal of, vol. 36, pp. 2003-2015, 2001.

[6] P. Shah (RF MAGIC INC (US)), "Harmonic Suppression Mixer and Tuner", patent WO2005091493 - 2005-09-29.

[7] R.C.H. van de Beek, D. M. W. Leenaerts, G. van der Weide, "A fasthopping single-PLL 3-band MB-OFDM UWB synthesizer", Solid-State Circuits, IEEE Journal of, Vol. 41, pp. 1522-1529, July 2006. 